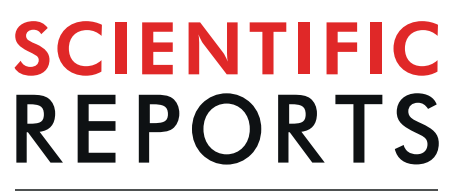

natureresearch

D) Check for updates

\title{
OPEN Author Correction: Relationship between spectrotemporal modulation detection and music perception in normal-hearing, hearing-impaired, and cochlear implant listeners
}

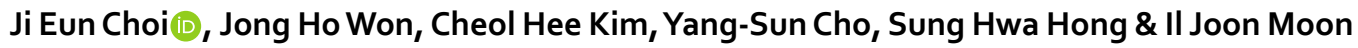

Correction to: Scientific Reports https://doi.org/10.1038/s41598-017-17350-w, published online 15 January 2018

The original version of this Article contained errors.

Ji Eun Choi and Cheol Hee Kim were incorrectly affiliated with 'Department of Otorhinolaryngology - Head and Neck Surgery, Samsung Medical Center, Sungkyunkwan University School of Medicine, Seoul, Republic of Korea'.

Affiliations 4 and 5 were omitted from the HTML and PDF versions of the Article.

Additionally, Affiliation 4 was incorrectly given in the Supplementary Information as 'Department of Otorhinolaryngology - Head and Neck Surgery, Samsung Medical Center, Sungkyunkwan University School of Medicine, Seoul, Republic of Korea'.

Furthermore, Affiliation 5 was incorrectly given in the Supplementary Information as 'Department of Otorhinolaryngology - Head and Neck Surgery, Samsung Changwon Hospital, Sungkyunkwan University School of Medicine, Seoul, Republic of Korea'

The correct author affiliations are listed below:

Affiliation 1:

Department of Otorhinolaryngology - Head and Neck Surgery, Dankook University Hospital, Cheoan, 31116, Republic of Korea

Ji Eun Choi

Affiliation 2:

Division of Ophthalmic and Ear, Nose and Throat Devices, Office of Device Evaluation, Center for Devices and Radiological Health, US Food and Drug Administration, Silver Spring, Maryland, 20993, United States

Jong Ho Won

Affiliation 3:

Hearing Research Laboratory, Samsung Medical Center, Seoul, 06351, Republic of Korea

Cheol Hee Kim 
Affiliation 4:

Department of Otorhinolaryngology-Head and Neck Surgery, Samsung Medical Center, Sungkyunkwan University School of Medicine, Seoul, 06351, Republic of Korea

Yang-Sun Cho \& Il Joon Moon

Affiliation 5:

Department of Otorhinolaryngology-Head and Neck Surgery, Samsung Changwon Hospital, Sungkyunkwan University School of Medicine, Changwon, 51353, Republic of Korea

\section{Sung Hwa Hong}

Finally, the Abstract contained a typographical error where,

“Ten normal-nearing (NH) listeners, ten hearing aid (HA) users with moderate hearing loss, and ten cochlear Implant (CI) users participated in this study."

now reads:

“Ten normal-hearing (NH) listeners, ten hearing aid (HA) users with moderate hearing loss, and ten cochlear Implant (CI) users participated in this study."

These errors have now been corrected in the PDF and HTML versions of the Article.

(c) (i) Open Access This article is licensed under a Creative Commons Attribution 4.0 International License, which permits use, sharing, adaptation, distribution and reproduction in any medium or format, as long as you give appropriate credit to the original author(s) and the source, provide a link to the Creative Commons license, and indicate if changes were made. The images or other third party material in this article are included in the article's Creative Commons license, unless indicated otherwise in a credit line to the material. If material is not included in the article's Creative Commons license and your intended use is not permitted by statutory regulation or exceeds the permitted use, you will need to obtain permission directly from the copyright holder. To view a copy of this license, visit http://creativecommons.org/licenses/by/4.0/.

(C) The Author(s) 2020 